\title{
Mitteilungen
}

\section{Psychodrama-Zentrum Münster}

Einführungs- und Entscheidungsseminar der berufsbegleitenden 3-jährigen Weiterbildung zum/r Supervisor/in DGSv

mit Dr. Ingeborg Wegehaupt-Schneider und Dieter Niehoff

31.1.-2.2.2003 in Hamburg

Information und Anmeldung: Psychodrama-Zentrum Münster, Alte Schanze 46, 48159 Münster, Tel. 0251/213548, Fax: 0251/211071, eMail: info@psychodrama-zentrum.de

\section{Institut für Beratung und Supervision Aachen}

Seminare und Worksops

Kreativitäts- und Innovationsmanagement

8.-9.11.2002, Köln

Dr. Anke Nienkerke-Springer

Krisen- und Konfliktmanagement für systemische SupervisorInnen

11.-13.12.2002 und 24.-26.3.2003

Heidi Neumann-Wirsig, Wolfgang Acht, Georg Nebel

Moderations- und Kommunikationsmethoden

10.-11.1.2003, Köln

Andrea Heer, MA, Prof. Dr. Heinz J. Kersting

Werkstatt für systemische Methoden in Supervision und Organisationsberatung

7.-8.2.2003 Aachen

Barbara Vogel, MA., Georg Nebel

Beginn der Ausbildung systemische Organisationsberatung

21.6.2003 Aachen

Dipl.-Soz. Brigitte Kemper Bürger, Prof. Dr. Heinz J. Kersting, Dipl.-Soz.Arb. Georg Nebel, Prof. Dr. Hans-Christoph Vogel

Information und Anmeldung: Institut für Beratung und Supervision Aachen, Heckstr. 25, 52080 Aachen, Tel.: 0241/953309, Fax: 0241/554815, E-mail office@ibs-networld.de 